\title{
Dialisi e gravidanza: quali prospettive?
}

\author{
Monica Limardo, Giuseppe Pontoriero \\ S.C. Nefrologia e Dialisi ASST Lecco, Ospedale A. Manzoni, Lecco
}

\begin{abstract}
Dialysis and pregnancy: new perspectives?
Although pregnancy is rare in women on dialysis, recent studies show that intensification of dialysis regimens or nocturnal dialysis treatment may be associated with unexpected improvements in pregnancy outcomes.

Nonetheless management of pregnancy in dialysis remains very challenging. Thus it becomes necessary that nephrologists dealing with dialysed women of child-bearing age be aware of these data to assist their patients to make informed decisions, when a pregnancy event is planned or arises.

It would be useful to have a systematic and prospective collection of pregnancy data in dialysis in order to have a more solid knowledge for clinical counselling.
\end{abstract}

Keywords: Chronic kidney disease, Dialysis, Outcomes, Pregnancy

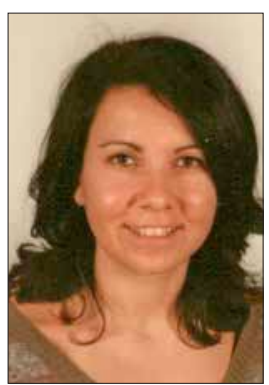

Monica Limardo
Sebbene la gravidanza rappresenti un evento eccezionale nelle donne in dialisi, recenti studi evidenziano come sia un evento meno raro rispetto a quanto atteso.

Le principali preoccupazioni riguardo alla gravidanza in dialisi sono legate all'aumentato rischio di preeclampsia, emergenze ipertensive e infezioni dovute agli accessi per la dialisi, alla prematurità e alla morte perinatale. Tali preoccupazioni sono legittime se consideriamo che, negli anni '70, solo il $23 \%$ delle gravidanze delle donne in dialisi si concludeva con un bambino nato vivo (1).

Dati più recenti sembrano mostrare un cambio di prospettive: in una recente analisi dei dati del Registro Australiano e Neozelandese dal 1966 al 2008 è stato osservato un incremento progressivo dell'incidenza della gravidanza in dialisi a partire dal 1996 (2). L'età mediana delle donne in gravidanza era pari a 30.7 anni, con una mediana di 19.6 mesi di durata

Accepted: May 11, 2016

Published online: May 30, 2016

Indirizzo per la corrispondenza:

Dr.ssa Monica Limardo

S.C. Nefrologia e Dialisi ASST Lecco

Ospedale A. Manzoni

Via Dell'Eremo 9/11

23900 Lecco

g.pontoriero@asst-lecco.it della dialisi prima del concepimento. Come atteso, il numero di complicanze è risultato elevato: il 19\% delle gravidanze era complicato da preeclampsia, la metà da parto pretermine e il $65 \%$ da un ridotto peso alla nascita. La mortalità perinatale è stata del $5 \%$. Significativo è il fatto che, escludendo le interruzioni volontarie di gravidanza, il $79 \%$ delle gravidanze si è concluso con un bambino nato vivo.

I progressi delle cure materno-neonatali degli ultimi decenni hanno avuto un importante impatto sugli outcome. In particolare, sul miglioramento degli outcome materno-fetali in dialisi, sembrano giocare un ruolo fondamentale l'intensificazione del programma dialitico e lo stretto follow-up clinico.

Un'analisi di confronto tra i dati del "Toronto Pregnancy and Kidney Disease Clinic and Registry" (2000-2013) e I'“American Registry for Pregnancy in Dialysis Patients" (1990-2011) (3) ha messo in luce una correlazione dose-risposta tra numero di ore settimanali di dialisi e outcome, in termini di tasso di natalità, durata della gravidanza e peso alla nascita: mentre il tasso di natalità era solo del $48 \%$ nelle donne trattate per meno di 20 ore settimanali di dialisi, gli outcome migliori sono stati osservati nelle donne trattate per più di 36 ore settimanali, con un tasso di natalità dell' $85 \%$.

Le donne canadesi, sottoposte a un trattamento dialitico intensivo, presentavano una durata mediana della gravidanza di 36 settimane, significativamente superiore $(p=0.002)$ rispetto alla mediana di 27 settimane osservata nelle donne americane in trattamento dialitico per $17 \pm 5$ ore settimanali. Anche la frequenza e la gravità delle complicanze maternofetali si riducevano nelle donne trattate per più di 36 ore settimanali e tali complicanze risultavano gestibili, secondo quanto riportato da Hladunewich et al (3).

Vi è una serie di meccanismi che potrebbero essere impli- 
cati nel miglioramento degli outcome nelle donne trattate più intensivamente: dalla riduzione della pressione arteriosa ai possibili effetti di miglioramento della funzione endoteliale e alla riduzione dell'infiammazione. I dati al momento disponibili suggeriscono che il successo dei regimi intensivi di dialisi sembra essere direttamente legato a una maggiore clearance dell'urea e, verosimilmente, anche degli altri soluti (4).

Oltre all'incremento della dose di dialisi, la gestione della gravidanza nel corso della dialisi è molto complessa e pone di fronte a delle sfide del tutto peculiari, tra cui l'adeguamento continuo della prescrizione dialitica in base ai cambiamenti che fisiologicamente avvengono in gravidanza, come l'aumento del volume ematico e della gittata cardiaca, la riduzione delle resistenze vascolari periferiche, l'anemia da emodiluizione, l'incremento di peso e le variazioni nel fabbisogno di calcio e fosforo, oltre che di vitamine e oligoelementi. La pressione deve essere attentamente controllata al fine di evitare sia l'ipertensione che l'ipotensione, e distinguere tra insorgenza di preeclampsia e ipertensione può essere, talvolta, difficile. La gestione della gravidanza in dialisi richiede, dunque, la collaborazione di tutta l'equipe medica e infermieristica e una forte integrazione dell'attività clinica con ginecologi e ostetrici esperti nella gestione della gravidanza a rischio. Assai utili sono, inoltre, la valutazione e il follow-up di un nutrizionista.

In questo contesto assai delicato e complesso, in cui i dati della letteratura sono assai limitati, il Gruppo di Studio "Rene e Gravidanza" della Società Italiana di Nefrologia nel 2015 ha pubblicato le raccomandazioni sulla gestione della gravidanza in dialisi, che offrono una valida guida pratica nella gestione clinica (5).

In Italia, in base a un'indagine condotta in 393 Centri Dialisi per il periodo 2000-2012, il tasso di natalità tra le donne in dialisi è stimato essere 10 volte inferiore che tra le donne portatrici di trapianto renale e circa 100 volte inferiore rispetto a quello della popolazione generale (6).

Da questa survey, le gravidanze sono risultate distribuite su tutto il territorio nazionale italiano, con casi anche in piccoli Centri Dialisi (6). Sebbene i casi di gravidanza in dialisi riportati in letteratura siano in aumento, l'esperienza clinica è limitata, anche nei Centri Dialisi di riferimento.

Al Nefrologo spetta il delicato compito di fornire un counselling pre-concezionale equilibrato sulla gravidanza e sui suoi possibili outcome alle giovani donne in dialisi, informandole anche del fatto che la probabilità di un successo è maggiore dopo il trapianto renale. Ciò supporta la scelta di pianificare una gravidanza dopo il trapianto di rene, almeno nelle giovani donne con un'elevata probabilità di ricevere il trapianto.

Concludendo, sebbene siano gravati da un aumentato rischio di complicanze, gli outcome della gravidanza in dialisi possono essere migliorati grazie a un approccio basato sull'intensificazione del trattamento dialitico, cambiando, rispetto al passato, le prospettive sulle possibilità e sugli outcome. Sarebbe auspicabile avere una raccolta sistematica e prospettica dei dati per avere basi più solide su cui basare il counselling.

\section{Disclosures}

Financial support: No financial support was received for this submission.

Conflict of interest: The authors have no conflict of interest.

\section{Bibliografia}

1. Report from the Registration Commitee of the European Dialysis and Transplant Association: successful pregnancies in women treated by dialysis and kidney transplantation. $\mathrm{Br} J$ Obstet Gynaecol. 1980;87:839-45.

2. Shahir HK, Briggs N, Katsoulis J, Levidiotis V. An observational outcomes study from 1966-2008, examining pregnancy and neonatal outcomes from dialysed women using data frome the ANZDATA Registry. Nephrology. 2013;18;276-84.

3. Hladunewich MA, Hou $S$, Odutayo $A$, et al. Intensive hemodialysis associates with improved outcomes: a Canadian and United States cohort comparison. J Am Soc Nephrol. 2014;25;1103-9.

4. Asamiya $Y$, Otsubo S, Matsuda $Y$, et al. The importance of low blood urea nitrogen levels in preganat patients undergoing hemodialysis to optimize birth weight and gestational age. Kidney Int. 2009;75(11):1217-22.

5. Cabiddu G, Castellino S, Gernone G, et al. Best practice on pregnancy on dialysis: the Italian Study Group on Kideny and pregnancy. J Nephrol. 2015;28:279-88.

6. Piccoli GB, Cabiddu G, Daidone $G$, et al. The children of dialysis: live born babies from on-dialysis mothers in Italy an epidemiological perspective comparing dialysis, kidney transplantation and the overall population. Nephrol Dial Transplant. 2014;29:1578-86. 